\title{
Nanophotonic interferometric immunosensors for label-free and real-time monitoring of chemical contaminants in marine environment
}

B. Chocarro-Ruiz, S. Herranz, A. Fernández Gavela, L.

M. Lechuga

B. Chocarro-Ruiz, S. Herranz, A. Fernández Gavela, L. M. Lechuga, "Nanophotonic interferometric immunosensors for label-free and real-time monitoring of chemical contaminants in marine environment," Proc. SPIE 10215, Advanced Environmental, Chemical, and Biological Sensing Technologies XIV, 1021503 (3 May 2017); doi: 10.1117/12.2256982

SPIE Event: SPIE Commercial + Scientific Sensing and Imaging, 2017, Anaheim, California, United States 


\title{
Nanophotonic interferometric immunosensors for label-free and real- time monitoring of chemical contaminants in marine environment
}

\author{
B. Chocarro-Ruiz ${ }^{a}$, S. Herranz ${ }^{a}$, A. Fernández Gavela, L.M. Lechuga* \\ Nanobiosensors and Bioanalytical Applications Group. Catalan Institute of Nanoscience and \\ Nanotechnology (ICN2), CSIC, The Barcelona Institute of Science and Technology and CIBER- \\ BBN, Campus UAB, Ed-ICN2, 08193 Bellaterra, Barcelona, Spain.
}

${ }^{a}$ These authors contributed equally to this work.

\begin{abstract}
With the aim to prevent the oceans ecosystems degradation, there is an urgent need to develop portable sensing tools able to operate directly in the environment, avoiding the transportation of the ocean samples to analytical laboratories. To achieve this long-term objective, we describe here the work carried out to develop and characterize a multiplexed photonic immunosensor for the direct analysis of toxic chemical targets in marine samples. We have employed immunosensors based on photonic Bimodal Waveguide (BiMW) interferometric devices fabricated in silicon technologies combined with specific receptors and antibodies for the targeted chemical targets. Several procedures for the functionalization of the $\mathrm{Si}_{3} \mathrm{~N}_{4}$ sensor surfaces have been evaluated based on wet silanization methods and further covalent receptor immobilization. The developed immunosensors, based on competitive inhibition assays, show LODs at $\mu \mathrm{g} / \mathrm{L}$ or $\mathrm{ng} / \mathrm{L}$ levels, depending on the analyzed chemical target.
\end{abstract}

Keywords: Nanophotonics, interferometric biosensors, environmental monitoring, environmental pollutants.

\section{INTRODUCTION}

The oceans are a clear example of the detrimental effects of many anthropogenic activities. The degradation of the marine environment is a fact but its extent and degree of transformation is unknown. To improve the surveillance of our oceans, there is an urgent need to develop efficient monitoring tools for measuring high toxic pollutants directly in the ocean samples, without a prior preconditioning. Biosensors have proven to be portable and reliable analytical devices capable of monitoring different types of contaminants in various types of matrices in a fast and reproducible manner ${ }^{1-3}$.

In the frame of the European Project BRAAVOO (Biosensors, Reporters and Algal Autonomous Vessels for Ocean Operation) (www.braavoo.org) dedicated to the development of biosensors for real-time monitoring of chemical contaminants of anthropogenic origin in the marine environment and its integration into ships and buoys, our aim was the development of a nanoimmunosensor module based on silicon technology for the on-site analysis of sea pollutants. As pollutants of interest, several organic compounds of diverse origin and characteristics were selected: four anthropogenic contaminants (the organo-halogenated pentabromodiphenyl or BDE-47, the antibiotics Ampicillin and Tetracycline, and the antifouling paint booster biocide Irgarol 1051), and two marine biohazard toxins from algal blooms (Domoic acid, DA and Okadaic acid, OA). To achieve a specific and sensitive response, antibodies were selected as recognition element for each of the above mentioned pollutants. Due to the low-molecular weight of the chemical pollutants and in order to be able to detect each of them in a label-free scheme, we have used a competitive assay format. In a competitive format, the analyte or a derivative is immobilized on the previously silanized sensor surface (receptor layer). The sample containing the chemical target to be detected is incubated for a few minutes with a fixed quantity of the specific antibody and then flowed onto the sensor surface. The signal is obtained by the interaction of the remaining free antibody of the incubated solution (sample + antibody) with the receptor layer. Therefore, the signal is inversely proportional to the concentration of the chemical target in the sample. The bioreceptor layer is expected to be re-usable during various analysis cycles by regeneration of the sensor surface, i.e. by dissociating the receptor-antibody interaction without destroying the bioreceptor activity (see Figure 1).

Advanced Environmental, Chemical, and Biological Sensing Technologies XIV, edited by

Tuan Vo-Dinh, Robert A. Lieberman, Proc. of SPIE Vol. 10215, 1021503 · @ 2017 SPIE

CCC code: $0277-786 \mathrm{X} / 17 / \$ 18 \cdot$ doi: $10.1117 / 12.2256982$ 


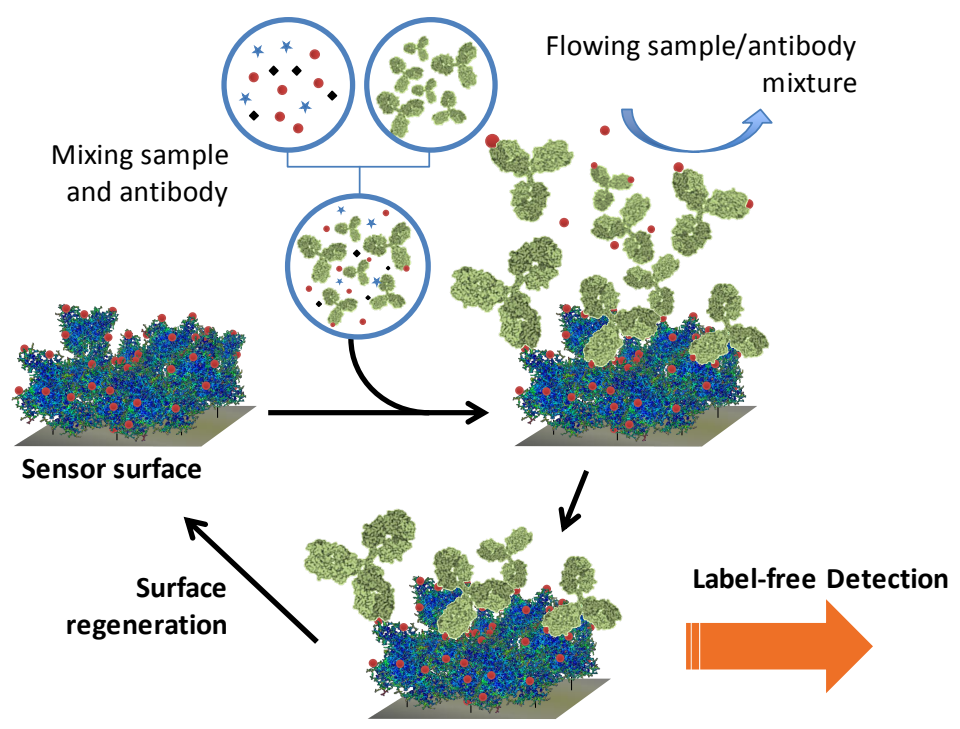

Figure 1. Competitive immunoassay format for label-free detection.

Specific receptor and antibodies for each of the six chemical targets were acquired commercially or through collaborations with other research groups or companies. Before the analysis with the BiMW nanointerferometric biosensors, we tested all the immunoreagents by custom-designed Surface Plasmon Resonance (SPR) biosensors. The sensitivity of the SPR biosensor for five of the six chemical targets was evaluated (data not shown). Only the BDE-47 immunosensor development was not evaluated, due to the poor response obtained with the available immunoreagents. The immunoassays for Irgarol 1051, Tetracycline, Ampicillin, Okadaic acid and Domoic acid detection were transferred from SPR to the development of immunosensors based on the photonic Bimodal Waveguide (BiMW) interferometric devices.

Although the BRAAVOO project is based on six different types of pollutants, this article will primarily focus on the BiMW biosensor performance for Okadaic acic detection as a proof of concept. The best responses were also obtained for the detection of the antibiotic tetracycline and the pesticide Irgarol $1051^{4}$, achieving limits of detection (LOD) at $\mu \mathrm{g} / \mathrm{L}$ or $\mathrm{ng} / \mathrm{L}$ level. Okadaic acid is a natural compound of the marine environment ${ }^{5}$. This is a type of compound produced by different species of microscopic algae, which can pass to mollusks and fish that feed on them, accumulating in the trophic chain, or released into the aquatic environment after death and lysis of the microorganism. They are a serious health problem especially when there are episodes of exponential growth of algal populations, difficult to predict. Ingestion of food contaminated with these substances (main route of human exposure) has different negative effects on human health ${ }^{6}$ : the main symptoms of OA poisoning are diarrhea, nausea, vomiting and abdominal pain. Other studies in animals have shown that OA can be a tumor promoter and proved its mutagenic and immunotoxic effects ${ }^{7}$.

\section{BIMODAL WAVEGUIDE INTERFEROMETRIC DEVICE}

The BiMW nanointerferometric devices have been described in our previous work ${ }^{8}$. In sum, the BiMW is a channel waveguide based on the interference of two propagation modes with the same polarization. First, the light is coupled in a rib-type waveguide that supports a fundamental mode. After some distance, this mode is coupled in a common path waveguide that supports two transverse modes, the fundamental and the first order ones. Both modes propagate to the chip output, where they create an interference pattern. In order to use the BiMW as a biosensor, a sensing window is opened in the top-cladding of the bimodal section. Since fundamental and first-order modes have different intensity distributions at the interface between the core and the cladding, the interference pattern at the output is a function of the refractive index in the sensing area. The BiMW sensor is based on silicon technology and fabricated at Clean Room facilities by standard microelectronics technology. Moreover, these type of photonic sensors based on integrated optics can be easily miniaturized and offer high potential for full integration and for multiplexed analysis. By the use of silicon photonic technology, additional advantages such as robustness, reliability, low power consumption and mass production potential are added, with the consequent reduction of future production costs. 
The performance of this biosensor has already been demonstrated for the detection of hormones ${ }^{9}$, bacteria ${ }^{10}$ or microRNA ${ }^{11}$ in real human samples (urine or ascetic fluid) showing excellent levels of sensitivity at the aM-pM level or at few cell per $\mathrm{mL}$.

\section{BIOFUNCTIONALIZATION PROTOCOLS}

Since the nanophotonic sensors have been developed using interferometric bimodal waveguides fabricated with silicon technology, the material of the waveguide is silicon nitride and the sensor area of the chip need to be biofunctionalized with the suitable biological receptor. The selected functionalization was the covalent immobilization using a previous silanization of the sensor surface. Starting with this well-established methodology, we have redesigned the strategy in order to: (i) increase the loading capacity of the immobilized biolayer, improving the sensitivity of the device, (ii) reduce or avoid the tendency to fouling of the sensing surface, allowing its application to the direct analysis of complex samples, (iii) increasing the stability of the immobilized bioreceptor, preserving its native structure during a high number of analysis cycles that will allow to reuse the nano-biosensor platform for multiple analyzes when placed in a real environment, and (iv) to ensure reproducibility of the biofunctionalization process between different sensor platforms.

The selected silanization is employing the carboxyethylsilanetriol sodium salt (CTES) that has been previously described by our Group ${ }^{12}$. In this case, the receptor can be directly immobilized before or after a blocking layer step. The protocol based on CTES silanization and further covalent receptor immobilization has been selected because it allows the successful receptor immobilization and it is one of the simplest and less time-consuming approaches.

In the case for OA, the chemical target was covalently attach to the sensor surface previously silanized with CTES and modified with a $70 \mathrm{kDa}$ amine-dextran network (Figure 2). Briefly, cleaned BiMW chips were silanized with CTES. An

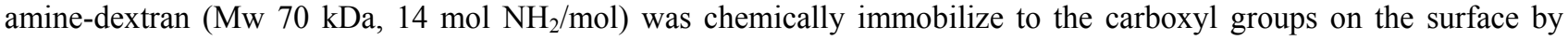
covering the chip sensing area with $100 \mu \mathrm{L}$ of an amine-dextran solution $(2.5 \mathrm{mg} / \mathrm{mL})$ in MES buffer $(100 \mathrm{mM} \mathrm{MES}$, $0.5 \mathrm{M} \mathrm{NaCl}, \mathrm{pH}$ 5.0) containing EDC $(0.2 \mathrm{M})$ and sulfo-NHS $(0.05 \mathrm{M})$ and left incubate for $4 \mathrm{~h}$ at $4{ }^{\circ} \mathrm{C}$. The chip was rinsed with deionized water and dried in a stream of $\mathrm{N}_{2}$. Afterwards, a mixture of $2 \mu \mathrm{L}$ of $2.5 \mathrm{mg} / \mathrm{mL}$ sulfo-NHS in MES buffer (100 mM MES, $0.5 \mathrm{M} \mathrm{NaCl}, \mathrm{pH} 5.0), 3 \mu \mathrm{L}$ of $17 \mathrm{mg} / \mathrm{mL}$ EDC in MES buffer (100 mM MES, $0.5 \mathrm{M} \mathrm{NaCl}, \mathrm{pH}$ $5.0)$ and $5 \mu \mathrm{L}$ of $1 \mathrm{mg} / \mathrm{mL}$ OA in DMSO was added to the chip surface and the chip was then covered with a glass-cover slip and placed in a petri dish with controlled moisture. The petri dish was covered with an aluminium foil and left in a refrigerator at $4{ }^{\circ} \mathrm{C}$ for $18 \mathrm{~h}$. Finally, the chip was rinsed with deionized water, dried with $\mathrm{N}_{2}$ and mounted in the BiMW platform.

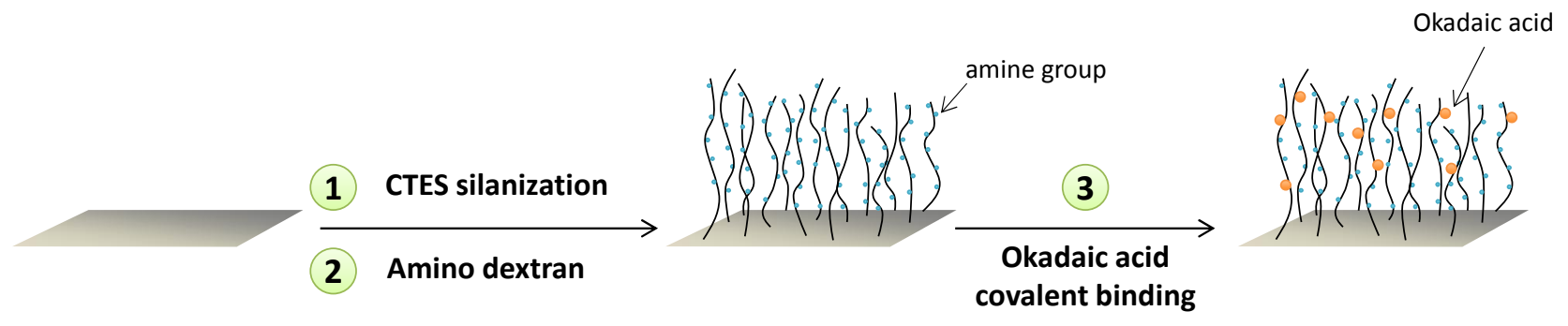

Figure 2. Covalent immobilization using okadaic acid as receptor onto the BiMW sensor chips

\section{PROOF OF CONCEPT OF THE BIMW SENSOR PERFORMANCE}

For OA immunosensor development, a commercial available anti-okadaic acid monoclonal antibody (anti-OA MAb) was purchased and the chemical target (OA) was used as receptor for the sensor surface functionalization, as previously mentioned. Before employing the BiMW, the selected antibody was evaluated using the SPR biosensor.

OA was covalently immobilized onto the gold surface previously modified with an amine-dextran network, as explained above. The effect of the amine-dextran network size used as crosslinking molecule was studied. Higher signal intensities were obtained when the size of the amine-dextran was increased from 10 to $70 \mathrm{kDa}$ (constant moles of amine group, see Figure 3). 


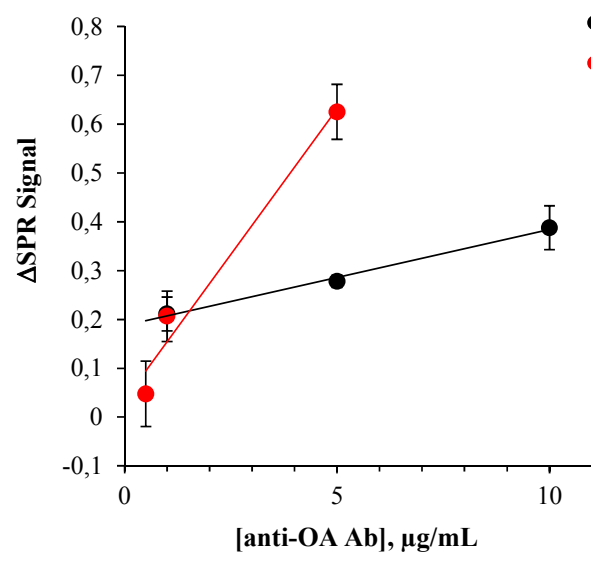

- $10 \mathrm{kDa}$ amine-dextran network

70 kDa amine-dextran network

Figure 3. SPR response for solutions of increasing anti-OA MAb concentration incubated onto (•) OAdextran(10kDa)-modified and (•) OA-dextran(70kDa)-modified sensing surface.

The response of an SPR chip modified with OA attached onto an amine-dextran (70kDa)-modified gold surface for solutions of increasing concentrations of OA was also evaluated as can be seen in Figure 4.

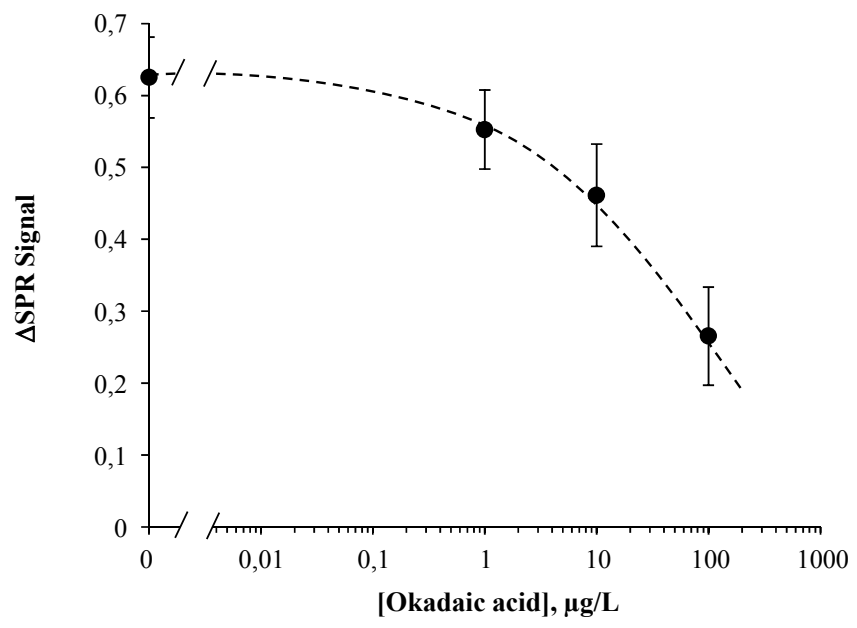

Figure 4. SPR response for solutions of increasing OA concentration (OA-dextran (70kDa)-modified gold chip; anti-OA MAb, $5 \mu \mathrm{g} / \mathrm{mL})$.

Results using the SPR biosensor showed an $\mathrm{IC}_{50}$ value and a LOD for OA of $\sim 50$ and $\sim 1 \mu \mathrm{g} / \mathrm{L}$, respectively. However, OA immobilization and immunoassay conditions could be further optimized in order to improve the sensor response and to reduce the antibody consumption. Since the aim of the project was to implement the BiMW biosensor, the optimization to achieve lower limits of detection and also a wider dynamic ranges, were carried out with the interferometric BiMW immunosensor.

The effect of the antibody concentration was evaluated with different anti-OA antibody concentration as can be seen in Figure 5. This is a critical parameter for the biosensor optimization, because the concentration needs to be high enough to achieve good sensitivity but still a limiting factor in such a way that the OA in the sample and the receptors onto the sensor surface compete for the binding sites of the antibodies. 


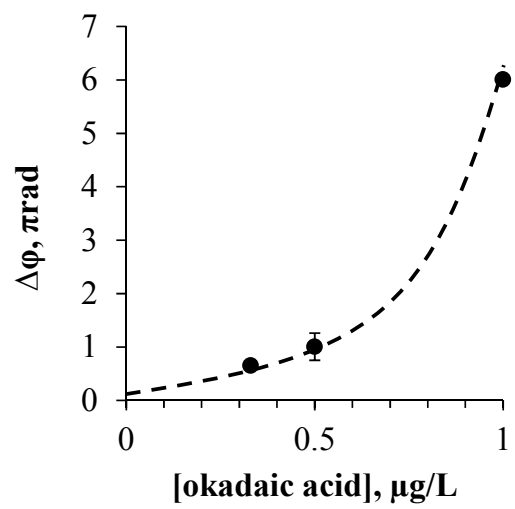

Figure 5. BiMW sesnor response for solutions of increasing anti-OA antibody concentrations.

By using an antibody concentration of $0.5 \mu \mathrm{g} / \mathrm{mL}$, the sensor response for OA standard solutions in the range from 0 to 1 $\mathrm{mg} / \mathrm{L}$ was evaluated (see Figure 6). Preliminary results show more sensitive analytical performance in the BiMW than for the SPR sensor, with a LOD of $0.2 \mu \mathrm{g} / \mathrm{L}$, an $\mathrm{IC}_{50}$ value of $1.3 \mu / \mathrm{L}$ and a dynamic range from 0.08 to $20 \mu \mathrm{g} / \mathrm{L}$. Moreover, the interaction between the immobilized OA and the anti-OA antibody can be dissociated by flowing onto the sensing channel a $100 \mathrm{mM} \mathrm{NaOH}$ solution for $120 \mathrm{~s}$, allowing regeneration and reusability of the functionalized surface for 9 measure-regeneration cycles.

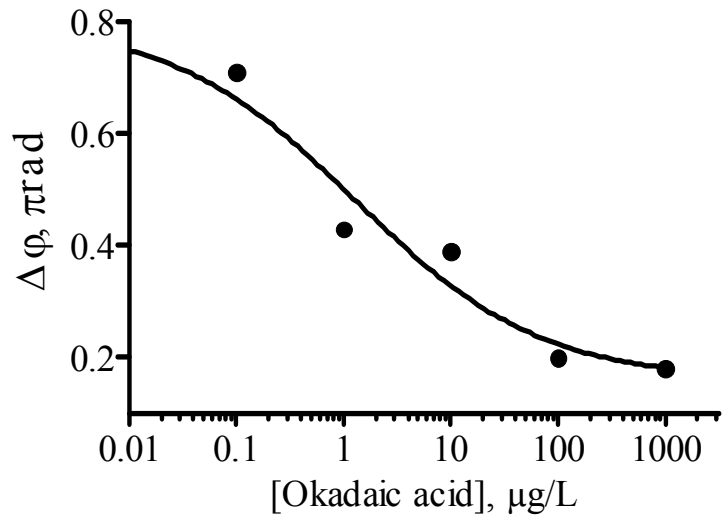

Figure 6. Calibration curve for Okadaic acid BiMW immunosensor (standard solutions prepared in PBS; anti$\mathrm{OA} \mathrm{Ab,} 0.5 \mu \mathrm{g} / \mathrm{mL})$.

\section{CONCLUSIONS}

The presented results show the promising performance of the BiMW interferometer biosensor for the detection of toxic pollutants in the marine environment. Preliminary results are shown in here for one of the target analytes, OA acid, showing a LOD of $0.2 \mu \mathrm{g} / \mathrm{L}$, an $\mathrm{IC}_{50}$ value of $1.3 \mu / \mathrm{L}$ and a dynamic range from 0.08 to $20 \mu \mathrm{g} / \mathrm{L}$. Although not shown in this manuscript, we have also obtained very promising results for the pesticide Irgarol $1051^{4}$. The LOD of the developed immunosensor for the detection of Irgarol 1051 in sea water is $3 \mathrm{ng} / \mathrm{L}$, so well below the $16 \mathrm{ng} / \mathrm{L}$ set by the European regulation as the maximum allowable concentration in sea water. Moreover, we have been working in the integration of the immunosensors with the microfluidics and the optical subsystem in order to provide an instrument ready to be incorporated in a stand-alone buoy.

Acknowledgements

This work has been funded by the 7FP (EU, BRAAVOO Grant Agreement No 614010). The nanoB2A is a consolidated research group (Grup de Recerca) of the Generalitat de Catalunya and has support from the Departament d'Universitats, 
Recerca i Societat de la Informació de la Generalitat de Catalunya (2014 SGR 624). ICN2 acknowledges support of the Spanish MINECO through the Severo Ochoa Centers of Excellence Program under Grant SEV-2013-0295. This work has made use of the Spanish ICTS Network MICRONANOFABS partially supported by MEINCOM.

\section{REFERENCES}

[1] D’Orazio, P., "Biosensors in clinical chemistry - 2011 update," Clin. Chim. Acta 412(19-20), 1749-1761 (2011).

[2] Zhang, W., Asiri, A. M., Liu, D., Du, D.., Lin, Y., "Nanomaterial-based biosensors for environmental and biological monitoring of organophosphorus pesticides and nerve agents," TrAC - Trends Anal. Chem. 54, 1-10 (2014).

[3] Fan, X., White, I. M., Shopova, S. I., Zhu, H., Suter, J. D.., Sun, Y., "Sensitive optical biosensors for unlabeled targets: A review," Anal. Chim. Acta 620(1-2), 8-26 (2008).

[4] B. Chocarro-Ruiz, S. Herranz, A. Fernández Gavela, J. Sanchís, M. Farréc, M.P. Marco, L. M. L., "Nanophotonic interferometric immunosensor for label-free and real-time monitoring of Irgarol 1051 in sea water," submitted (2017).

[5] Sassolas, A., Hayat, A., Catanante, G.., Marty, J. L., "Detection of the marine toxin okadaic acid: Assessing seafood safety," Talanta 105, 306-316 (2013).

[6] Tubaro, A., Sosa, S.., Hungerford, J., "Toxicology and diversity of marine toxins," [Veterinary Toxicology], Elsevier, 896-934 (2012).

[7] EFSA., "Opinion of the Scientific Panel on Contaminants in the Food Chain on a request from the European Commission related to pyrrolizidine alkaloids as undesiderable substances in animal feed," EFSA J. 2007(447), $1-51$ (2007).

[8] Zinoviev, K. E., González-Guerrero, A. B., Domínguez, C.., Lechuga, L. M., "Integrated bimodal waveguide interferometric biosensor for label-free analysis," J. Light. Technol. 29(13), 1926-1930, IEEE (2011).

[9] González-Guerrero, A. B., Maldonado, J., Dante, S., Grajales, D.., Lechuga, L. M., "Direct and label-free detection of the human growth hormone in urine by an ultrasensitive bimodal waveguide biosensor," J. Biophotonics 10(1), 61-67 (2016).

[10] Maldonado, J., González-Guerrero, A. B., Dom??nguez, C.., Lechuga, L. M., "Label-free bimodal waveguide immunosensor for rapid diagnosis of bacterial infections in cirrhotic patients," Biosens. Bioelectron. 85(447), 310-316 (2016).

[11] Huertas, C. S., Fariña, D.., Lechuga, L. M., "Direct and Label-Free Quantification of Micro-RNA-181a at Attomolar Level in Complex Media Using a Nanophotonic Biosensor," ACS Sensors 1(6), acssensors.6b00162 (2016).

[12] González-Guerrero, A. B., Alvarez, M., Castaño, A. G., Domínguez, C.., Lechuga, L. M., "A comparative study of in-flow and micro-patterning biofunctionalization protocols for nanophotonic silicon-based biosensors," J.

Colloid Interface Sci. 393, 402-410 (2013). 